SPECIAL MEETINGS 


\section{COMMISSION 17 \\ A. LUNAR PROBES}

(Saturday, August 26, 1967)

Chairman: Dr D. Menzel

Out of the programme, published in the I.A.U. Transactions XIIIB, the following contributions were selected for publication in the present volume:

1. I. Taback: A Description of the Lunar Orbiter Spacecraft.

2. W.E. Brunk: Photographic Results of the Lunar Orbiter Program. 


\title{
A DESCRIPTION OF THE LUNAR ORBITER SPACECRAFT
}

\author{
ISRAEL TABACK
}

\section{Introduction}

The U.S. Lunar Orbiter Spacecraft Program was conceived to search out potential Apollo landing sites, and to return detailed photographic coverage of the lunar surface for scientific study. The first flight was launched in August 1966, and the successful launch of the fifth orbiter was in August 1967. All five were successful and returned to Earth a large amount of photographic data.

Presented in this paper is a short description of the spacecraft system with particular emphasis on the photographic system. The intent is to supply information which will permit a better understanding of the photographic data to be presented by Dr. William Brunk in the following paper.

\section{Mission Plan}

Figure 1 schematically shows the launch and flight operations which were applied for all of the orbiters. During the launch phase, telemetry and tracking are accomplished by the Eastern Test Range (ETR) stations. At about the time the spacecraft

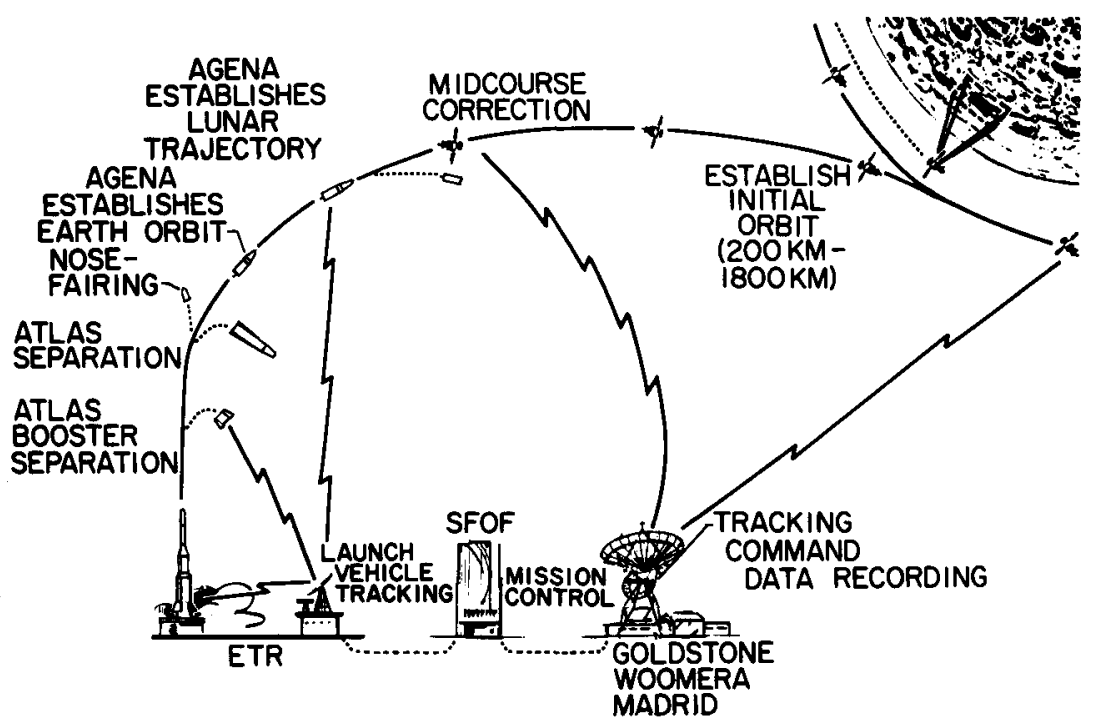

FIG. 1. Mission operations.

Perek (ed.), Highlights of Astronomy, 462-470. (C) I.A.U. 
is inserted into a translunar trajectory, tracking, telemetry, and command transmission is accomplished by the Goldstone (Nevada), Madrid (Spain), and Woomera (Australia) stations of the NASA Deep Space Instrumentation Facility (DSIF). Centralized control is accomplished from NASA Space Flight Operations Facility (SFOF) in Pasadena, Calif.

\section{Spacecraft Configuration}

Figure 2 shows the general configuration of the $390 \mathrm{~kg}$ ( 850 pound) spacecraft which is approximately $1 \frac{1}{2} \mathrm{~m}$ ( 5 feet) high and $1 \frac{1}{2} \mathrm{~m}$ in diameter, excluding the solar panels and antennas. The span across the deployed antenna booms is about $6 \mathrm{~m}$.

With the exception of the rocket engine and its fuel tanks, which are used for midcourse maneuvers and deboost into lunar orbit, essentially all of the major spacecraft components are attached to an equipment mounting plate. That mounting plate, the underside of which remains oriented toward the Sun at all times except when the spacecraft is maneuvering, provides thermal control for the components attached to it.

In the flight configuration, all of the main spacecraft structure above the equipment mounting plate is covered with a highly reflective shroud of aluminum-coated mylar which constitutes part of the thermal control system. The only protrusions through that shroud are the camera lenses and micrometeoroid detectors.

The spacecraft power system is a conventional solar array/storage battery type. A 12 amp-hour nickel cadmium battery is used to supply the spacecraft power requirements during the launch phase prior to solar array deployment and during those periods of the lunar orbit when the spacecraft is in the Moon's shadow. Because of the rather severe weight penalty for battery capacity, it has been imperative to mini-

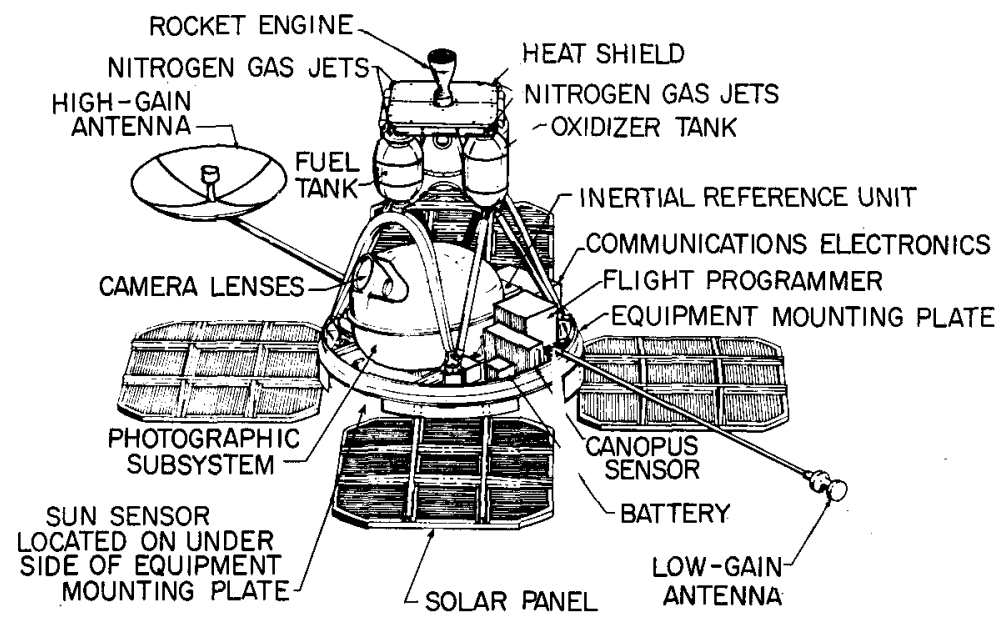

Fig. 2. Spacecraft configuration. 
mize the power consumption of the residual power load 5 , including such communication system items as the transponder, command decoder, etc. When the solar array is in sunlight, a maximum of about $375 \mathrm{~W}$ is available to handle all power demands including battery recharging. The voltage of the spacecraft dc-power bus can vary from a minimum of $22 \mathrm{~V}$ to a maximum of $31 \mathrm{~V}$ when the array is in operation. A shunt regulator is used to prevent the voltage from exceeding $31 \mathrm{~V}$.

The star Canopus and the Sun are the primary references for spacecraft attitude orientation. For maneuvering, or when those references are occulted, a strappeddown gyro system is used. Attitude control is accomplished by a cold-gas system.

A photograph of the camera system is shown in Figure 3. The spacecraft camera system employs two lenses (Figure 4), which take simultaneous pictures on a roll of $70-\mathrm{mm}$ wide aerial film. One of the lenses has a $610 \mathrm{~mm}(24 \mathrm{inch})$ focal length and can take pictures from an altitude of $46 \mathrm{~km}$ with a resolution of approximately $1 \mathrm{~m}$. The other lens, which has a focal length of about $80 \mathrm{~mm}$ ( 3 inches), takes pictures with a resolution of approximately $8 \mathrm{~m}$. The film is developed onboard using a method which presses the film into contact with a web that contains a single-solution processing chemical. After the film has been dried, it is ready for read-out and transmission to Earth.

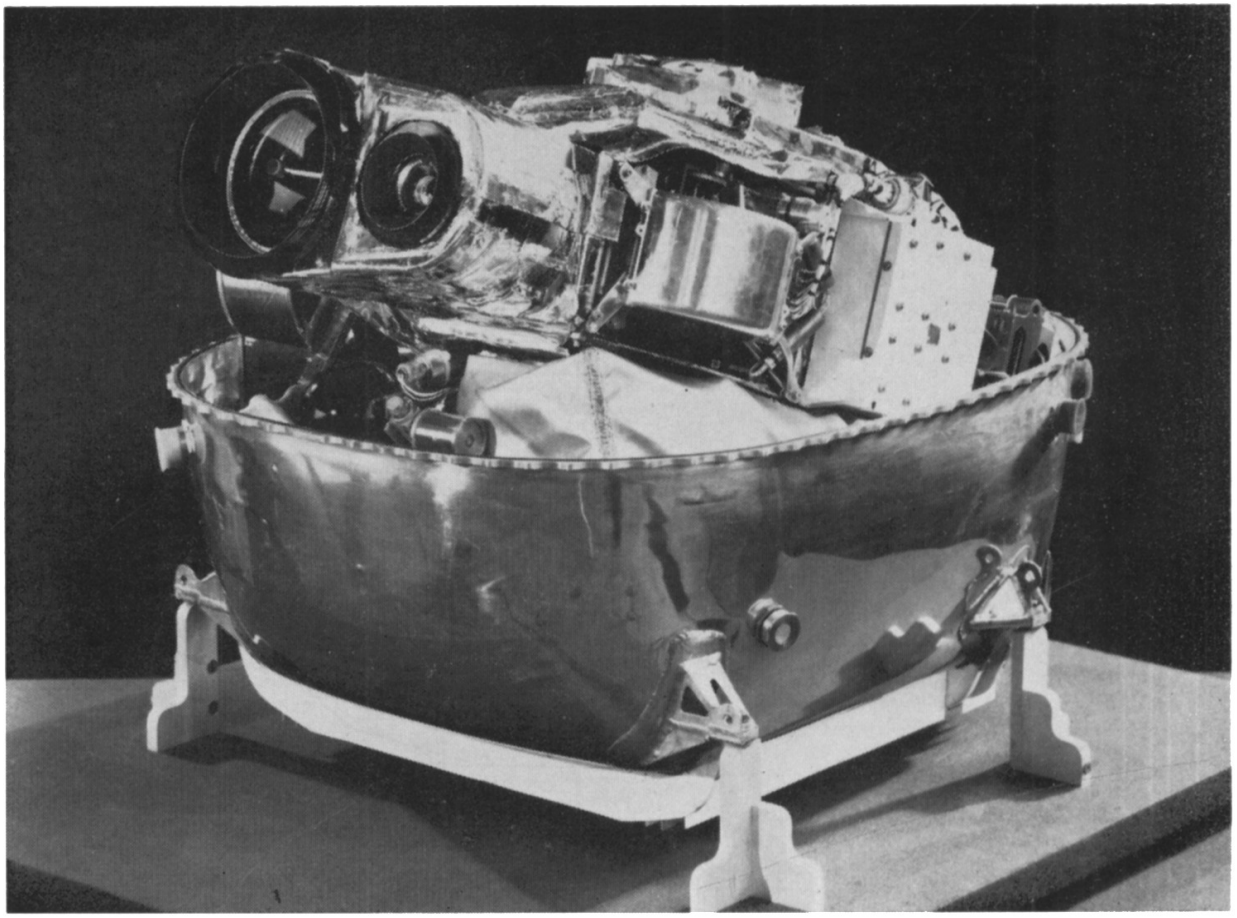

FIG. 3. Camera subsystem. 


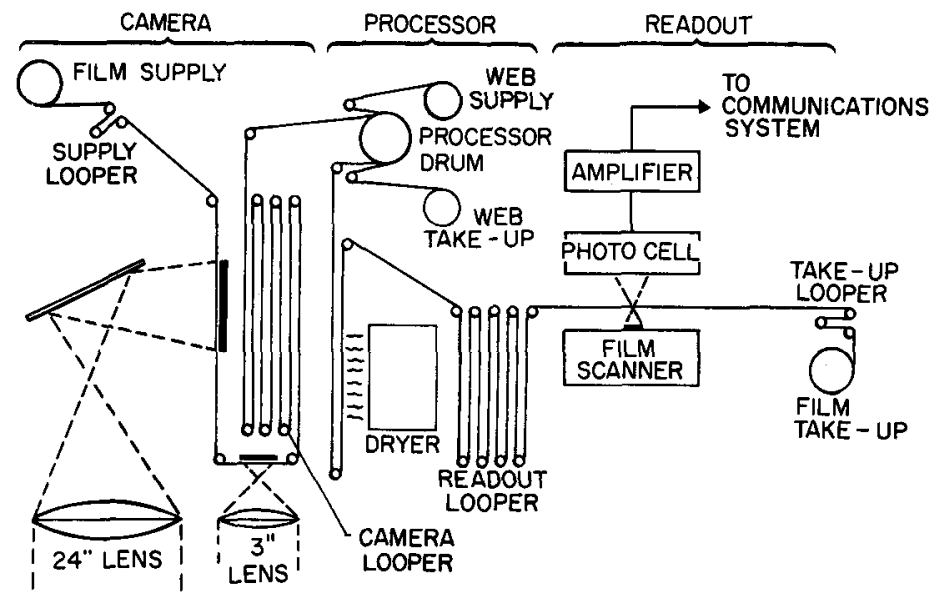

FIG. 4. Spacecraft photographic system.

Figure 5 is a drawing of the read-out system which uses a line-scan tube as the light source for film scanning. The line-scan tube itself electronically scans the beam of light through an excursion of $2.5 \mathrm{~mm}(0 \cdot 1 \mathrm{inch})$ in a direction parallel to the film travel. Sweep of the light beam in the other direction (across the width of the film) is accomplished by a mechanically driven optical system, which also serves to reduce the diameter of the beam of light to approximately 5 microns. In read-out, this type of scan produces a series of 'framelets' which are $2.5 \mathrm{~mm}$ in one direction and the width of the film in the other direction. As the figure shows, scanning begins at one edge of the film and continues across the film until the opposite edge is reached. At that time the film is advanced and is scanned in the opposite direction. This sequence is repeated, with the scan rates being such that $43 \mathrm{~min}$ are required to scan about $28 \mathrm{~cm}$ (1 foot) of film which contains one high-resolution and one medium-resolution photograph.

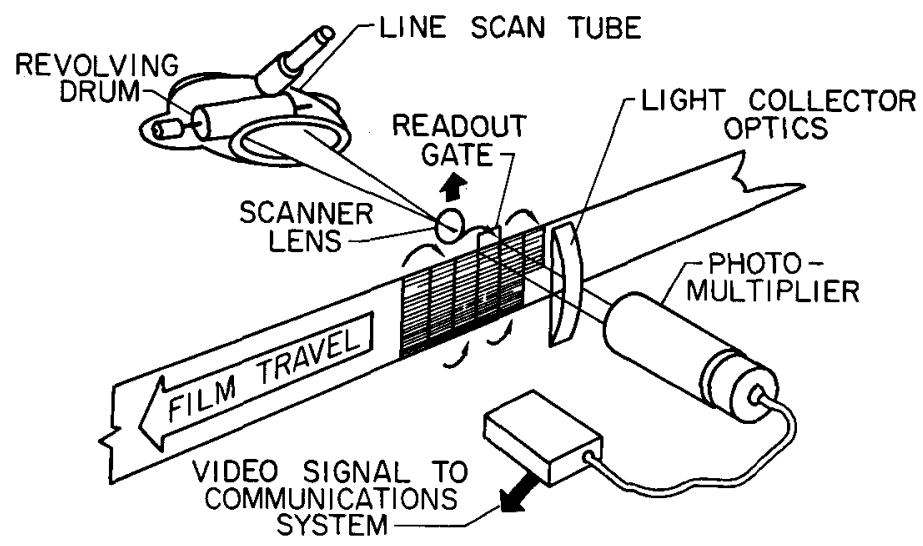

FIG. 5. Photographic system read-out schematic. 
Collecting optics direct the transmitted light into a photomultiplier, and the resulting electrical signal is conditioned and mixed with synch and blanking pulses and fed to the communication system modulator.

Each spacecraft carries a film supply of about $85 \mathrm{~m}$ (260 feet), which is sufficient to photograph, from an altitude of $46 \mathrm{~km}, 12000 \mathrm{~km}^{2}$ of lunar surface with a resolution of $1 \mathrm{~m}$ and $200000 \mathrm{~km}^{2}$ with a resolution of $8 \mathrm{~m}$. It is interesting to note that about one million standard commercial TV pictures would be required to photograph that area with comparable resolution.

Figure 6 indicates the modes of picture taking. The moderate-resolution and highresolution fields of view have a common centre. Pictures may be taken at a rate which

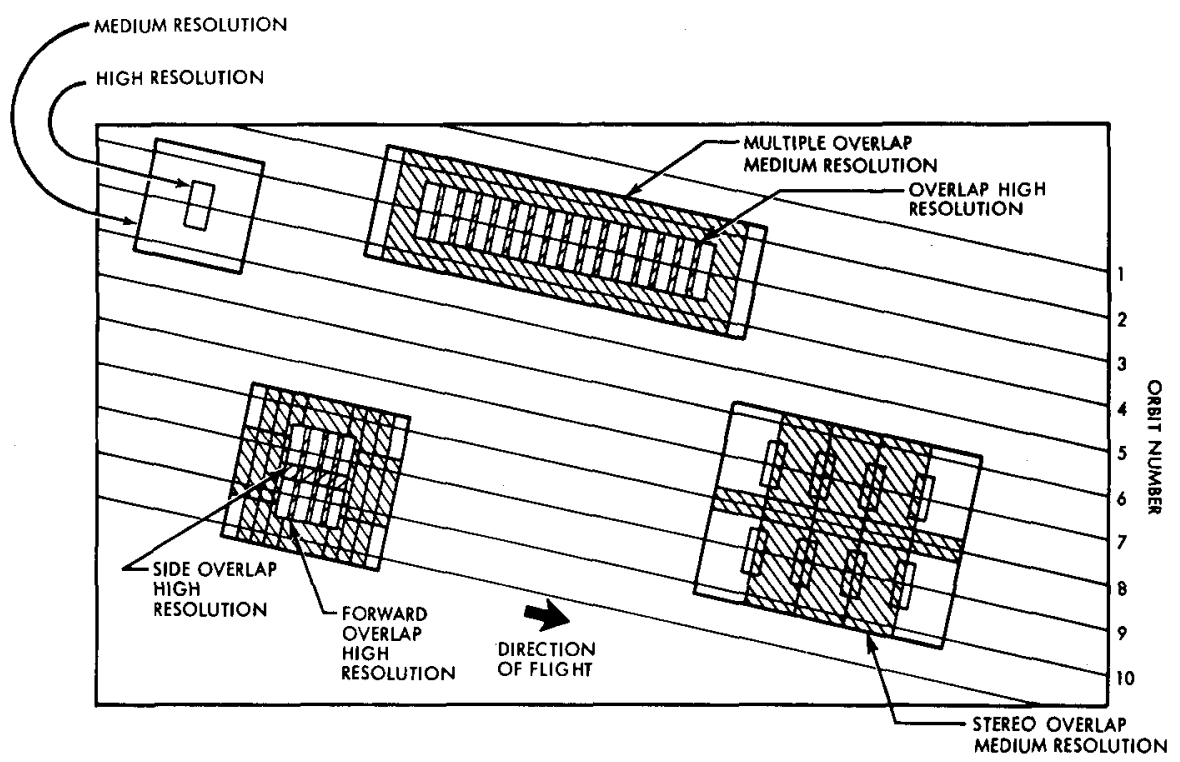

FIG. 6. Photographic modes.

results in contiguous high-resolution coverage, or at a rate which provides stereo coverage with only samples of spaced high-resolution photography. Stereo coverage was also obtained on some of the flights by providing side-overlap of photography with a proper base to height ratio.

\section{Orbital Sequence}

Figure 7 shows the sequence of orbits secured by the five missions. The first three were at low inclinations, primarily to secure coverage of the near-equatorial Apollo sites. On these three flights, 8 sites were located and vertical stereo and oblique photography obtained. Flight four resulted in photography which essentially covered 


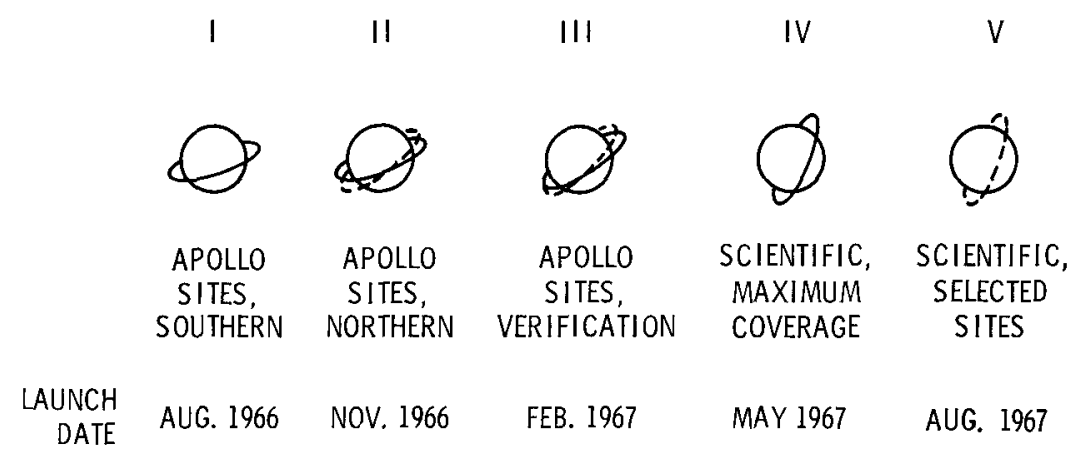

Fig. 7. Lunar Orbiter missions.

the entire front face of the Moon at resolutions of at least one order of magnitude greater than previously available from Earth-based photography. The result, a mosaic which covers a large portion of a gymnasium floor, is available for those who wish to walk on the Moon. The fifth flight covered over 40 sites of particular scientific interest at a resolution of a few meters, and completed coverage of the far side of the Moon.

Table 1 is a tabulation of the orbital parameters for each flight and indicates the accuracy with which these orbits were established at the Moon. These photographic orbits were, in general, those secured after first establishing a somewhat coarser orbit, and then making an orbital correction to secure proper perilune altitude, period, and orbit inclination. The apolunes and perilunes are in kilometers and the orbit inclination is given in degrees.

Table 1

\section{Final orbit parameters}

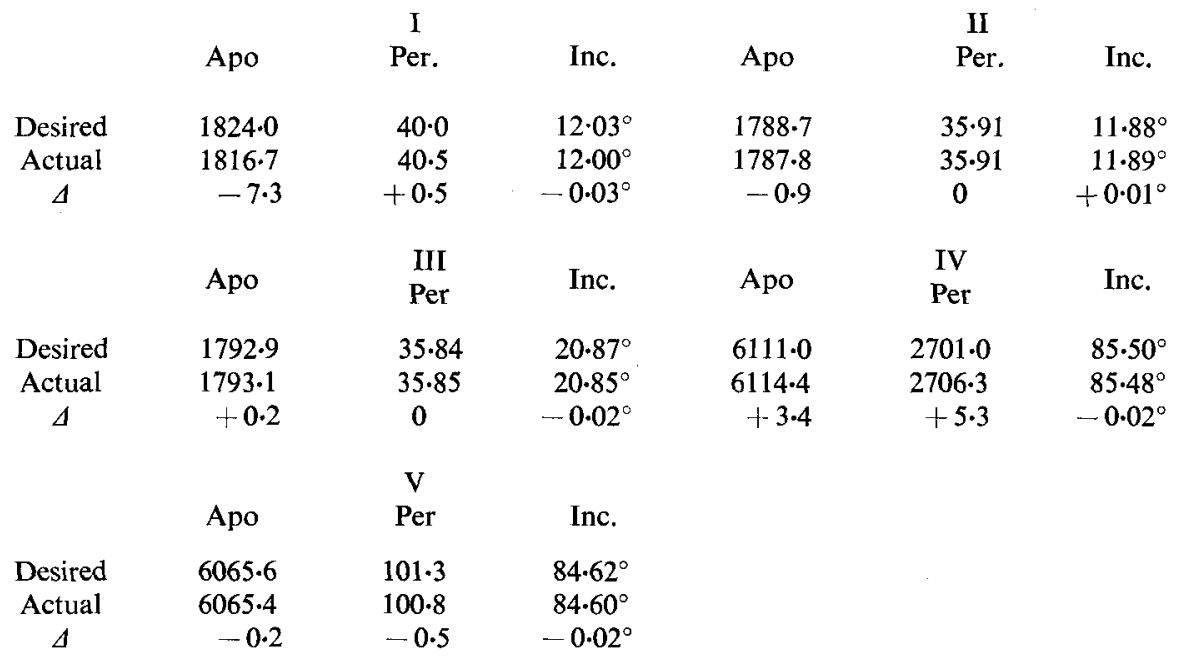




\section{Results}

The orbiters returned a considerable quantity of data from the Moon, not all of which was photographic. Table 2 lists the data secured.

Continuous tracking of the orbiters has resulted in a definition of many of the higher harmonics of the gravitational field. This work has been reported by Michael and Tolson (1965).

\section{Table 2}

\section{Information return}

1. Information for Apollo Sites

2. Information on Surface Properties

3. General Geological Information

4. Special Interest Photographs

5. Non-photographic
(a) Selenodesy
(b) Micrometeorite Flux
(c) Radiation Flux
(d) Surface Height (V/H Sensor)

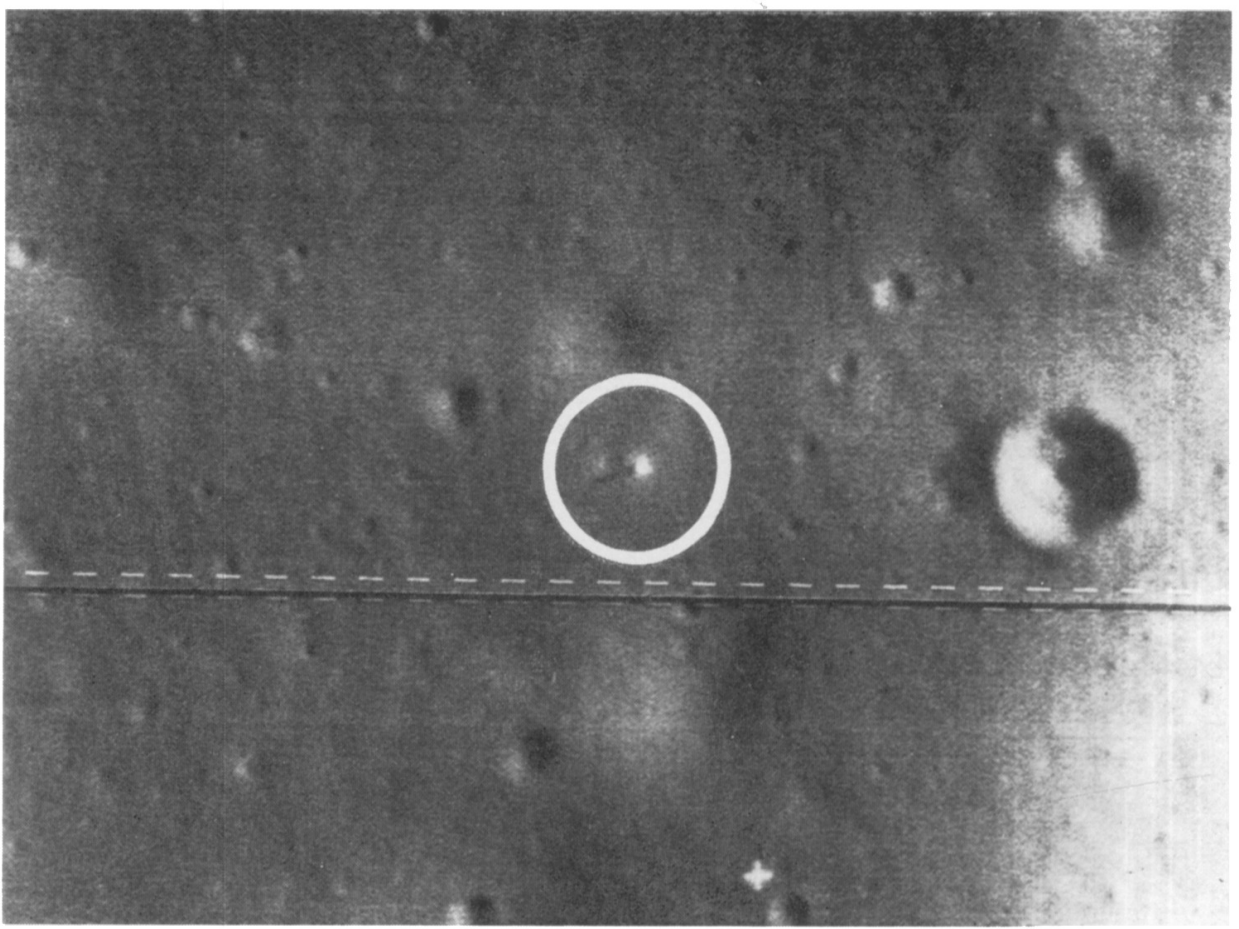

FIG. 8. Landed Surveyor. 
The micrometeoroid detectors, 20 pressurized cans on each spacecraft, have been punctured at a rate which to date averages approximately $\frac{1}{3}$ of the rate which has been measured in the near-Earth environment. Data are still being collected from the three operating spacecraft which are still in orbit.

The radiation detectors have provided measurements during solar flares indicating that the environment near the Moon is similar to that of deep space.

\section{Selected Photographs}

Only two pictures will be shown in this paper, which are indicative of the fact that the photography secured provides some information regarding lunar surface properties.

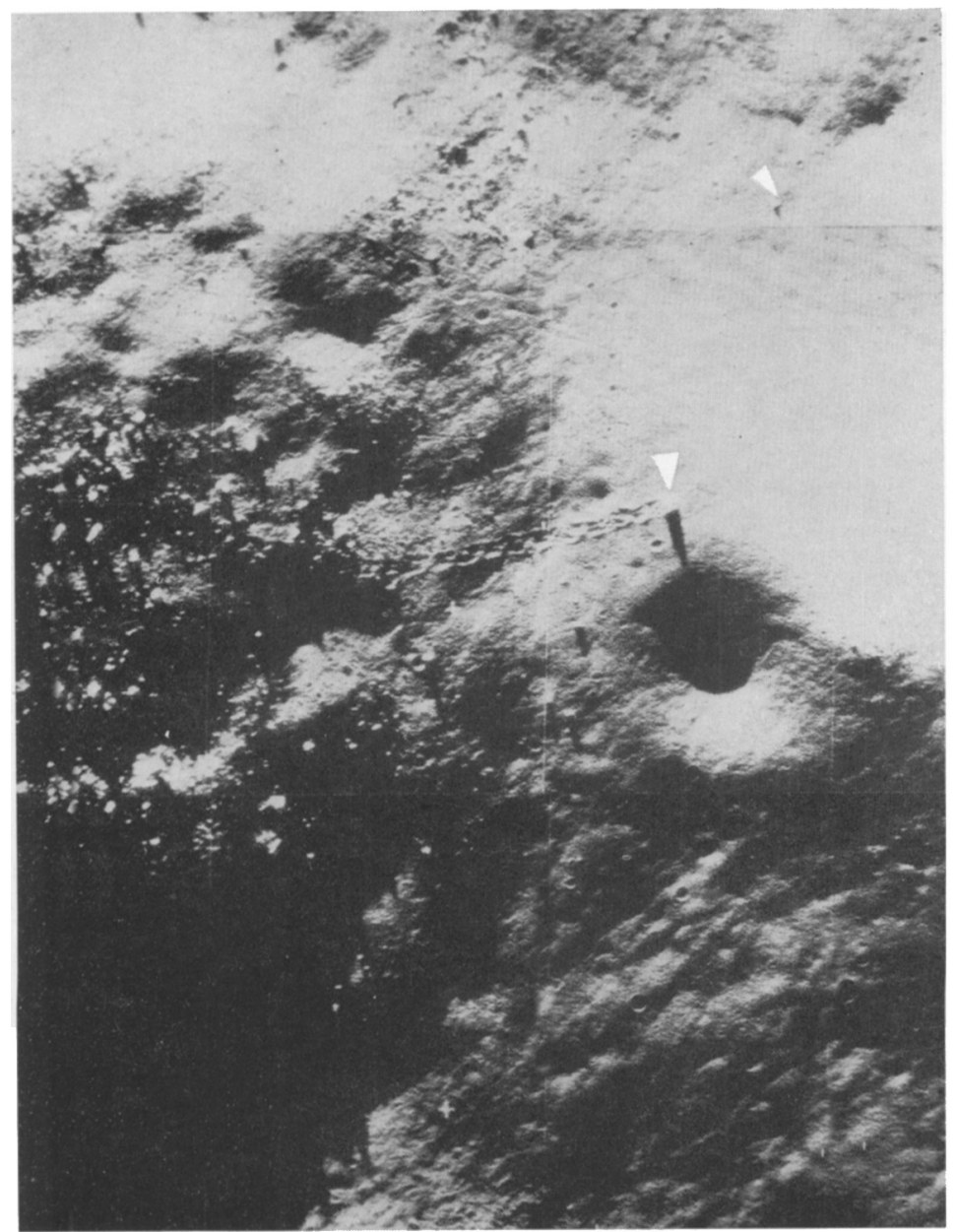

FIG. 9. 'Rolling Stones'. 
Figure 8 is a picture taken of Surveyor-I by Lunar Orbiter-II. A positive identification was made by correlating numerous objects in the Surveyor picture with the same objects in the orbiter picture. This identification allowed extrapolation of Surveyor results over a wide area.

Figure 9 shows the tracks of two boulders which have rolled down an incline and are still visible. By making estimates of the boulder density it is possible to draw some conclusions regarding the bearing properties of the lunar surface.

In conclusion it can be stated that the Lunar Orbiters have provided not only the specific information required for manned exploration, but have secured photographic coverage of the entire lunar surface at a resolution never before attained.

\section{References}

Kosofsky, Leon J., Broome, G. Calvin (1965) Lunar Orbiter: A Photographic Satellite. Presented at Society of Motion Picture and Television Engrs. (Los Angeles, Calif.), Mar. 28-Apr. 2.

Michael, William H., Jr., Tolson, Robert H. (1965) The Lunar Orbiter Project Selenodesy Experiment. NASA paper presented at the Second International Symposium on the Use of Artificial Satellites for Geodesy (Athens, Greece), Apr. 27-May 1.

Taback, Israel (1964) Lunar Orbiter: Its Mission and Capability. Preprint 64-7, Am. Astronaut. Soc., May 1964.

Taback, I., Brummer, E.A. (1965) The Lunar Orbiter. Presented at AIAA Unmanned Spacecraft Meeting (Los Angeles, Calif.), Mar. 1-3. 